\title{
Review \\ Year in review 2005: Critical Care - Respirology: mechanical ventilation, infection, monitoring, and education
}

Jack J Haitsma ${ }^{1}$, Jesús Villar ${ }^{1,2}$ and Arthur S Slutsky ${ }^{1}$

1'Interdepartmental Division of Critical Care, University of Toronto, Toronto, Canada
${ }^{2}$ Canarian Institute for Biomedical Research, Canary Islands, Spain

Corresponding author: Arthur S Slutsky, arthur.slutsky@utoronto.ca

Published: 29 June 2006

Critical Care 2006, 10:217 (doi:10.1186/cc4959)

This article is online at http://ccforum.com/content/10/3/217

(c) 2006 BioMed Central Ltd

\begin{abstract}
We summarize all original research in the field of respiratory intensive care medicine published in 2005 in Critical Care. Twenty-seven articles were grouped into the following categories and subcategories to facilitate rapid overview: mechanical ventilation (physiology, spontaneous breathing during mechanical ventilation, high frequency oscillatory ventilation, side effects of mechanical ventilation, sedation, and prone positioning); infection (pneumonia and sepsis); monitoring (ventilatory monitoring, pulmonary artery catheter and pulse oxymeter); and education (training and health outcome).
\end{abstract}

\section{Introduction}

This article summarizes the original research in the field of respirology that was published in 2005 in Critical Care. We grouped the articles into subcategories to help the reader get a rapid overview of the key articles and thus focus on topics of interest.

\section{Mechanical ventilation Physiology}

In acute respiratory distress syndrome (ARDS) the amount of aerated lung is markedly reduced as a result of alveolar collapse and flooding. Rylander and coworkers [1] assessed the volume of poorly or nonventilated gas in mechanically ventilated ARDS patients. They assumed that the difference ratio between ventilated gas volume (end-expiratory lung volume, measured using sulphur hexafluoride) and total gas volume (total end-expiratory gas volume independent of ventilation, measured using computed tomography) represents poorly or nonventilated gas volume. About one-third of the total gas volume was poorly or nonventilated in the lungs of ARDS patients at a positive end-expiratory pressure (PEEP) level of $5 \mathrm{cmH}_{2} \mathrm{O}$. This uneven distribution of ventilation is probably due to the presence of small airway closure and/or obstruction, and a recruitment procedure might ameliorate this.

To detect recruitment, Henzler and coworkers [2] compared commonly used measures of lung mechanics for improvement in oxygenation. In an easily recruitable model of acute lung injury (ALI), recruitment $\left(45 \mathrm{cmH}_{2} \mathrm{O}\right.$ for $\left.40 \mathrm{~s}\right)$ did not result in oxygenation changes consistent with recruitment. However, changes in aerated and nonaerated lung after the recruitment manoeuvre were adequately represented by changes in plateau pressure, respiratory system compliance and recruitable volume. It seems that the degree of improvement in oxygenation is not so much determined by reduction in nonaerated lung as by the blood flow through these regions.

Extracorporeal membrane oxygenation (ECMO) provides temporary extracorporeal life support for children with severe respiratory or cardiac failure. ECMO therapy is discontinued when adequate pulmonary and/or cardiac function returns. As oxygenation improves, tidal volume increases and chest radiographs reveal a reduction in pulmonary opacification. Hermon and coworkers [3] investigated whether surfactant application could influence these variables in children with respiratory failure. After application of surfactant, mean tidal volume almost doubled (186\% of baseline value), mean compliance increased significantly (176\% of baseline value) and radiographic scores tended to decrease within 48 hours. Surfactant treatment did not affect mortality but it helped in weaning from ECMO.

Spontaneous breathing during mechanical ventilation Wrigge and colleagues [4] demonstrated that spontaneous breathing with airway pressure release ventilation promotes

$\mathrm{ALI}=$ acute lung injury; $\mathrm{ARDS}=$ acute respiratory distress syndrome; $\mathrm{CMV}=$ conventional mechanical ventilation; $\mathrm{COPD}=$ chronic obstructive pulmonary disease; $\mathrm{ECMO}=$ extracorporeal membrane oxygenation; $\mathrm{ET}=$ endothelin; HFOV = high-frequency oscillatory ventilation; IAP $=$ intraabdominal pressure; ICU = intensive care unit; IPAH = idiopathic pulmonary arterial hypertension; IPV = intrapulmonary percussive ventilation; PCP = pulmonary capillary pressure; PEEP = positive end-expiratory pressure; SARS = severe acute respiratory syndrome; VAT $=$ ventilator-associated tracheobronchitis. 
alveolar recruitment mainly in dependent, juxtadiaphragmatic lung regions, resulting in end-expiratory aeration. It also led to a redistribution of tidal ventilation to dependent lung zones in oleic acid induced lung injury. Spontaneous breathing with airway pressure release ventilation countered the undesirable cyclic alveolar collapse in dependent lung regions, which can contribute to ventilator-associated lung injury, but no indices of lung injury were assessed. These data are in support of the hypothesis that active contractions of the diaphragm are a major explanatory factor in the improved oxygenation observed in animal models and in patients with ALI with reduction in intrapulmonary shunt. These data suggest that allowing spontaneous breathing activity in ALI could help to reduce lung injury.

Haberthür and Guttmann [5] repeatedly applied a pattern of different levels of PEEP in a heterogeneous population of tracheally intubated patients during unsupported spontaneous breathing. The level of PEEP significantly influenced resting short-term breathing patterns by selectively affecting the duration of expiration. A reduction in PEEP was paralleled by an increase in respiratory rate and in minute ventilation, and paradoxically - tidal volume and inspiratory time were not significantly affected. These data suggest the existence of the Hering-Breuer inflation/deflation reflex in adults under standard clinical conditions; this reflex, produced by increased stretch receptor activity, leads to a prolongation of expiration.

\section{High-frequency oscillatory ventilation}

Bollen and coworkers [6] reported the results of a multicentre, multinational trial of the safety and efficacy of highfrequency oscillatory ventilation (HFOV) in ARDS patients. The study was prematurely stopped because of poor patient accrual and the completion of a similar trial. Thirty-seven patients received HFOV and 24 patients received conventional mechanical ventilation (CMV). There were no statistically significant differences in mortality or survival without supplemental oxygen. The authors suggested that future trials should incorporate the oxygenation index (given by [fractional inspired oxygen $x$ mean airway pressure $\times$ 100]/arterial carbon dioxide tension) for more informative analysis, because a post hoc analysis suggested that there was a relative improvement with HFOV compared with CMV in patients with a higher baseline oxygenation index.

Slee-Wijffels and colleagues [7], in a retrospective single centre study, reported on the use of HFOV in paediatric patients $(n=53)$ who deteriorated on CMV. They observed that HFOV rescue therapy was associated with a high overall survival (64\%). In patients with diffuse alveolar disease ( $n=32$ ) the survival rate was $56 \%$, and this rate was $88 \%$ in patients with small airway disease $(n=17)$. Future studies are necessary to evaluate whether the outcome in patients with diffuse alveolar disease may be improved if HFOV is applied earlier in the course of the lung disease.
Intrapulmonary percussive ventilation (IPV), a high-frequency ventilation technique that is intended for therapeutic mobilization of bronchial secretions, has primarily been used in patients with cystic fibrosis in a stable state. However, it has shown promise for increasing sputum production in patients with chronic obstructive pulmonary disease (COPD). Vargas and coworkers [8], in a randomized trial conducted in patients with exacerbation of COPD, demonstrated that 30 min of IPV led to a significant decrease in respiratory rate, an increase in arterial oxygen tension and a decrease in arterial carbon dioxide tension $(P<0.05)$. No worsening of exacerbation was observed in patients treated with IPV, as compared with deterioration in six out of 17 patients in the control group $(P<0.05)$. The hospital stay was also significantly shorter in the IPV group than in the control group (6.8 \pm 1.0 days versus $7.9 \pm 1.3$ days; $P<0.05)$.

\section{Side effects of mechanical ventilation}

Pneumothorax often complicates the management of patients with severe acute respiratory syndrome (SARS) who are ventilated. Kao and coworkers [9] concluded that SARS patients who suffered pneumothorax (12\% of patients) were more tachypnoeic on admission, and had more pronounced hypoxaemia and hypercapnia during hospitalization, although there were no significant differences in pressure, volume, or mortality rate between patients with and those without pneumothorax.

Critically ill patients frequently develop anaemia during their intensive care unit (ICU) stay, which has partly been attributed to a blunted erythropoietin response [10]. Erythropoietin is an endogenous glycoprotein hormone that is the primary stimulus for erythropoiesis. DeAngelo and colleagues [10] demonstrated that the erythropoietin response is also blunted in anaemic (haemoglobin $10.6 \mathrm{~g} / \mathrm{dl}$ ) mechanically ventilated patients compared with matched anaemic ambulatory patients.

\section{Sedation}

Breen and coworkers [11] reported a randomized, openlabel, multicentre study comparing the safety and efficacy of an analgesia-based sedation regimen using remifentanil ( $n=57$ ) with those of a midazolam-based sedation regimen with fentanyl or morphine added as analgesia $(n=48)$ in critically ill patients requiring prolonged mechanical ventilation for up to 10 days. The remifentanil-based sedation regimen reduced the duration of mechanical ventilation by 53.5 hours $(P=0.033)$ and reduced the time from the start of the weaning process to extubation by 26.6 hours $(P<0.001)$.

\section{Prone positioning}

Prone positioning has been shown to improve arterial oxygenation in about two-thirds of patients with ARDS; however, it also increases intra-abdominal pressure (IAP). The increased IAP does not severely impair cardiopulmonary, renal, or hepatosplanchnic functions during short periods of prone positioning. However, many patients included in 
previous studies were placed on air-cushioned beds, which might have had an impact on these variables. In a prospective randomized crossover study, Michelet and coworkers [12,13] compared prone positioning on an air-cushioned bed with that on a conventional foam mattress. Although IAP was significantly higher and liver function decreased on the conventional mattress, these effects were not associated with differences in oxygenation or cardiovascular parameters.

\section{Infection}

\section{Pneumonia}

Mortensen and colleagues [14] investigated the impact of the empiric use of $\beta$-lactams and fluorquinolones in severe pneumonia. The use of only $\beta$-lactams has been reported to be associated with increased mortality, whereas the combination with macrolides improved outcome. In a retrospective analysis of 172 eligible patients (community-acquired pneumonia) over a 3-year period, Mortensen and colleagues [14] observed a 30-day mortality of $30 \%$ in patients treated with $\beta$-lactams and fluorquinolone $(n=50)$ as compared with a rate of $17.2 \%$ in patients treated with other guidelineconcordant antibiotics ( $n=87)$. After adjusting for confounders, the use of $\beta$-lactam and fluoroquinolone was associated with increased mortality (odds ratio 2.71 ).

In patients with cystic fibrosis aerosolized colistin has successfully been used to treat acute pulmonary exacerbations of infection or initial colonization with Pseudomonas aeruginosa strains. Michalapoulos and coworkers [15] retrospectively identified eight patients with nosocomial pneumonia who received aerosolized colistin. Survival and clinical cure rates for the infection were better in patients with pneumonia who received additional aerosolized colistin, although these findings were not statistically significantly different from those in patients who received only intravenous colistin treatment (survival $7 / 8$ patients [87.5\%] versus 34/45 patients [75.6\%], $P=0.41$; clinical cure: $7 / 8$ patients [87.5\%] versus $30 / 45$ patients [66.7\%], $P=0.67)$. The number of patients included was very small, but this retrospective study suggests that there is a need for a randomized controlled trial examining the efficacy and safety of aerosolized colistin for the management of patients with nosocomial pneumonia.

Grigoriu and colleagues [16] conducted a prospective study in mechanically ventilated patients and demonstrated that cytological lesions of alveolar damage, expressed as the presence of haemorrhage and desquamated type II pneumocytes in bronchoalveolar lavage fluid, can be found in patients with severe hospital-acquired or community-acquired pneumonia. However, the poor sensitivity and the necessity for a trained pathologist limits the potential utility of this approach for day-to-day practice.

Ventilator-associated tracheobronchitis (VAT) is a common nosocomial infection among mechanically ventilated patients, with incidence rates reported in the literature of 3.7-10.6\%. Investigating the outcome of VAT in a retrospective, observational, matched study design in patients without chronic respiratory failure, Nseir and colleagues [17] found that both duration of mechanical ventilation and ICU stay were prolonged in these patients, although there was no difference in mortality. Although all patients with VAT were treated with antibiotics and treated for a longer period than matched control individuals, most were not treated specifically for VAT. Analysis of adequacy of antibiotic treatment failed to show any difference, although this result is limited by the small number of patients receiving adequate treatment.

Garnacho-Montero and coworkers [18] assessed risk factors, clinical features, management and outcomes in critically ill patients in whom Aspergillus spp. were isolated from respiratory secretions, using a database of 1756 patients from a study designed to assess fungal infections. Treatment with steroids (odds ratio 4.5), COPD (odds ratio 2.9) and neutropenia (odds ratio 9.4) were significantly associated with Aspergillus spp. isolation. Aspergillus spp. was recovered in 36 patients; in 14 patients isolation of Aspergillus spp. was interpreted as colonization (mortality 50\%), in 20 patients it was interpreted as invasive aspergillosis (mortality 80\%), and two cases were not classified. Antifungal treatment should be considered in patients with clinical features of pneumonia, with isolation of Aspergillus spp. from respiratory secretions, and who have either COPD or are undergoing treatment with corticosteroids. In contrast, antifungal treatment should not be initiated when Aspergillus spp. are recovered from bronchial aspirates of critically ill patients without predisposing risk factors and in the absence of clinical and radiological signs of pneumonia. In these cases, isolation of Aspergillus spp. should be interpreted as colonization.

\section{Sepsis}

In sepsis and ARDS a cascade of inflammatory responses leads to production of proinflammatory cytokines. This cascade might be inhibited by eliminating the pathogenic toxins using haemoperfusion with a polymyxin B immobilized fibre column. In a prospective uncontrolled observational study, Kushi and coworkers [19] used this technique in 36 patients with ARDS caused by sepsis. Haemoperfusion reduced levels of plasminogen activator inhibitor-1, neutrophil elastase and interleukin- 8 in blood at 48 hours, and improved arterial oxygen tension/fractional inspired oxygen ratio at 96 hours after the start of treatment.

During sepsis, endothelin (ET)-1 is released into the bloodstream by endothelial cells causing local vasoconstriction, especially in the pulmonary circulation. Blocking the ET-1 receptor decreases the extravascular lung water index during endotoxaemia [20]. Kuklin and coworkers [20] reported a possible mechanism for explaining how a nonselective ET-1 receptor blocker (tezosentan) reduces translocation of the $\alpha$ - 
isoform of protein kinase $\mathrm{C}$ from the cytosol to the membrane during endotoxaemia. In similar experiments, conducted in an experimental animal model in which pulmonary oedema develops following sepsis caused by caecal ligation and puncture, Kuklin and coworkers [21] reduced pulmonary oedema by blocking the ET-1 receptor. These experiments suggest a possible way to reduce lung injury during sepsis.

\section{Monitoring Ventilatory monitoring}

Frank and coworkers [22] used the Dräger Oxylog 3000 ${ }^{\mathrm{TM}}$ (Dräger Medical, Best, The Netherlands) to demonstrate that oscillatory flow with potentially harmful effects may occur during ventilation, especially in conditions with high resistance such as occur in children with narrowed airways (endotracheal tube internal diameter $<6 \mathrm{~mm}$ ) or severe obstructive lung disease or airway diseases in adult patients. If oscillations are present, then the ventilator setting must be adjusted by reducing the steepness of the ramp in biphasic intermittent positive airway pressure and assisted spontaneous breathing, or by reducing the tidal volume in pressure-limited intermittent positive pressure ventilation.

A partial carbon dioxide rebreathing technique can be used to estimate cardiac output in mechanically ventilated patients. However, carbon dioxide rebreathing can result in increased minute ventilation and an irregular respiratory pattern. Tachibana and coworkers [23] reported the use of a shorter duration of rebreathing ( $35 \mathrm{~s}$ versus $50 \mathrm{~s}$ ), which mitigated these effects but yielded a cardiac output measurement that is similar in accuracy.

\section{Pulmonary artery catheters}

Pulmonary capillary pressure (PCP) is the major force determining fluid filtration from pulmonary capillaries into the interstitium, and thus it is a major determinant of oedema formation. Souza and coworkers analyzed pulmonary artery pressure decay curves in patients with pulmonary hypertension due to either idiopathic pulmonary arterial hypertension (IPAH; $n=12)$ or ARDS $(n=11)$ [24]. Using a mathematical analysis based on fitting these curves, they demonstrated that PCP in IPAH patients is greater than normal, and that the different algorithms yielded different PCP within the groups. The time needed to reach steady-state pulmonary arterial occlusion pressure was longer in the IPAH group (higher time constants). However, methodological limitations to this occlusion technique confound interpretation. Different disease processes may result in different times for arterial emptying, with resulting implications for the methods available for estimating PCP.

\section{Pulse oxymetry}

Respiratory variation in arterial pulse pressure has been suggested as a predictor of fluid responsiveness in mechanically ventilated patients with circulatory failure. The main limitation of this method is that it requires an invasive arterial catheter. Cannesson and coworkers [25], in a prospective clinical trial in 22 mechanically ventilated patients, demonstrated a strong correlation between respiratory variation in arterial pulse pressure and respiratory variation in pulse oximetry plethysmographic waveform amplitude. Although this was a limited study, a respiratory variation in pulse oximetry plethysmographic waveform amplitude above $15 \%$ accurately discriminated between patients with a respiratory variation in systemic pulse pressure above $13 \%$ from those with a variation of $13 \%$ or less. The study suggests that this may be a noninvasive approach to assessment of fluid responsiveness in ventilated patients.

Wang and coworkers [26] reported the novel approach of using a pulse oximeter to monitor left main bronchus $\mathrm{SpO}_{2}$ by attaching it to a double lumen tube. $\mathrm{SpO}_{2 \text { trachea }}$ was the same as $\mathrm{SvO}_{2 \text { blood }}$ in haemodynamically stable animals as well as during haemorrhagic shock, suggesting that this technique would be reliable both in the operating theatre and in ICU patients; as such it may be a possible alternative to the pulmonary artery catheter for certain measurements.

\section{Education}

\section{Training}

Abrahamson and coworkers [27] described the effective implementation of a simulation protocol during SARS to train health care workers in managing cardiac arrest while wearing unfamiliar equipment and following a modified advanced cardiac life support protocol. Simulation provided insights that had not been considered in earlier phases of development of the protocol. It was used to detect and correct flaws and omissions in a protocol specific to treatment of SARS patients. The insights gained from this experience will be valuable for future infectious disease challenges in the ICU.

\section{Health outcomes}

Needham and coworkers [28] describe the Improving Care of ALI Patients (ICAP) study, a multisite, prospective cohort study that aims to evaluate longer term outcomes of ALI/ARDS survivors, with a particular focus on the effect of low tidal volume ventilation and other critical care therapies. The study is enrolling consecutive mechanically ventilated ALI/ARDS patients from $11 \mathrm{ICUs}$ at four hospitals in the city of Baltimore, Maryland, USA, with an expected enrollment of 520 patients over a 2-year period. Exposures (patient based, clinical management, and ICU organizational) will be comprehensively collected both at baseline and throughout the patients' ICU stays. Outcomes, including mortality, organ impairment, functional status and quality of life, will be assessed with the use of standardized surveys and testing at 3, 6, 12 and 24 months after ALI/ARDS diagnosis. A multifaceted retention strategy will be used to minimize participant loss to follow up.

\section{Competing interests}

AS has received consulting income from BOC, GSK, Hamilton Medical, $\mathrm{KCl}$, Maquet and Ventana. AS has also 
been paid for being a member of the following Data Safety and Monitoring Boards: Asthmatx, Broncus and Leo Pharma.

\section{References}

1. Rylander C, Tylen U, Rossi-Norrlund R, Herrmann P, Quintel M, Bake $B$ : Uneven distribution of ventilation in acute respiratory distress syndrome. Crit Care 2005, 9:R165-R171.

2. Henzler D, Pelosi P, Dembinski R, Ullmann A, Mahnken AH, Rossaint $R$, Kuhlen R: Respiratory compliance but not gas exchange correlates with changes in lung aeration after a recruitment maneuver: an experimental study in pigs with saline lavage lung injury. Crit Care 2005, 9:R471-R482.

3. Hermon M, Burda G, Male C, Boigner H, Ponhold W, Khoss A, Strohmaier W, Trittenwein G: Surfactant application during extracorporeal membrane oxygenation improves lung volume and pulmonary mechanics in children with respiratory failure. Crit Care 2005, 9:R718-R724.

4. Wrigge $H$, Zinserling J, Neumann $P$, Muders $T$, Magnusson A, Putensen C, Hedenstierna G: Spontaneous breathing with airway pressure release ventilation favors ventilation in dependent lung regions and counters cyclic alveolar collapse in oleic-acid-induced lung injury: a randomized controlled computed tomography trial. Crit Care 2005, 9:R780-R789.

5. Haberthur C, Guttmann J: Short-term effects of positive endexpiratory pressure on breathing pattern: an interventional study in adult intensive care patients. Crit Care 2005, 9:R407R415.

6. Bollen CW, van Well GT, Sherry T, Beale RJ, Shah S, Findlay G, Monchi M, Chiche JD, Weiler N, Uiterwaal CS, et al:: High frequency oscillatory ventilation compared with conventional mechanical ventilation in adult respiratory distress syndrome: a randomized controlled trial [ISRCTN24242669]. Crit Care 2005, 9:R430-R439.

7. Slee-Wijffels FY, van der Vaart KR, Twisk JW, Markhorst DG, Plotz FB: High-frequency oscillatory ventilation in children: a single-center experience of $\mathbf{5 3}$ cases. Crit Care 2005, 9:R274R279.

8. Vargas F, Bui HN, Boyer A, Salmi LR, Gbikpi-Benissan G, Guenard H, Gruson D, Hilbert G: Intrapulmonary percussive ventilation in acute exacerbations of COPD patients with mild respiratory acidosis: a randomized controlled trial [ISRCTN17802078]. Crit Care 2005, 9:R382-R389.

9. Kao HK, Wang JH, Sung CS, Huang YC, Lien TC: Pneumothorax and mortality in the mechanically ventilated SARS patients: a prospective clinical study. Crit Care 2005, 9:R440R445.

10. DeAngelo AJ, Bell DG, Quinn MW, Long DE, Ouellette DR: Erythropoietin response in critically ill mechanically ventilated patients: a prospective observational study. Crit Care 2005, 9: R172-R176.

11. Breen D, Karabinis A, Malbrain M, Morais R, Albrecht S, Jarnvig IL, Parkinson P, Kirkham AJ: Decreased duration of mechanical ventilation when comparing analgesia-based sedation using remifentanil with standard hypnotic-based sedation for up to 10 days in intensive care unit patients: a randomised trial [ISRCTN47583497]. Crit Care 2005, 9:R200-R210.

12. Michelet $P$, Roch A, Gainnier M, Sainty JM, Auffray JP, Papazian L: Correction: Influence of support on intra-abdominal pressure, hepatic kinetics of indocyanine green and extravascular lung water during prone positioning in patients with ARDS: a randomized crossover study. Crit Care 2005, 9:308.

13. Michelet $P$, Roch A, Gainnier M, Sainty JM, Auffray JP, Papazian L: Influence of support on intra-abdominal pressure, hepatic kinetics of indocyanine green and extravascular lung water during prone positioning in patients with ARDS: a randomized crossover study. Crit Care 2005, 9:R251-R257.

14. Mortensen EM, Restrepo MI, Anzueto A, Pugh J: The impact of empiric antimicrobial therapy with a beta-lactam and fluoroquinolone on mortality for patients hospitalized with severe pneumonia. Crit Care 2005, 10:R8.

15. Michalopoulos A, Kasiakou SK, Mastora Z, Rellos K, Kapaskelis AM, Falagas ME: Aerosolized colistin for the treatment of nosocomial pneumonia due to multidrug-resistant Gram-negative bacteria in patients without cystic fibrosis. Crit Care 2005, 9:R53-R59.
16. Grigoriu B, Jacobs F, Beuzen F, El Khoury R, Axler O, Brivet FG, Capron F: Bronchoalveolar lavage cytological alveolar damage in patients with severe pneumonia. Crit Care 2005, 10:R2.

17. Nseir S, Di Pompeo C, Soubrier S, Lenci H, Delour P, Onimus T, Saulnier F, Mathieu D, Durocher A: Effect of ventilator-associated tracheobronchitis on outcome in patients without chronic respiratory failure: a case-control study. Crit Care 2005, 9:R238-R245.

18. Garnacho-Montero J, Amaya-Villar R, Ortiz-Leyba C, Leon C Alvarez-Lerma F, Nolla-Salas J, Iruretagoyena JR, Barcenilla F: Isolation of Aspergillus spp. from the respiratory tract in critically ill patients: risk factors, clinical presentation and outcome. Crit Care 2005, 9:R191-R199.

19. Kushi H, Miki T, Okamaoto K, Nakahara J, Saito T, Tanjoh K: Early hemoperfusion with an immobilized polymyxin B fiber column eliminates humoral mediators and improves pulmonary oxygenation. Crit Care 2005, 9:R653-R661.

20. Kuklin V, Kirov M, Sovershaev M, Andreasen T, Ingebretsen OC, Ytrehus K, Bjertnaes L: Tezosentan-induced attenuation of lung injury in endotoxemic sheep is associated with reduced activation of protein kinase C. Crit Care 2005, 9:R211-R217.

21. Kuklin V, Sovershaev M, Andreasen T, Skogen V, Ytrehus K, Bjertnaes L: Tezosentan reduces the microvascular filtration coefficient in isolated lungs from rats subjected to cecum ligation and puncture. Crit Care 2005, 9:R677-R686.

22. Frank GE, Trimmel H, Fitzgerald RD: Inspiratory oscillatory flow with a portable ventilator: a bench study. Crit Care 2005, 9: R315-R322.

23. Tachibana K, Imanaka H, Takeuchi M, Nishida T, Takauchi $Y$, Nishimura M: Effects of reduced rebreathing time, in spontaneously breathing patients, on respiratory effort and accuracy in cardiac output measurement when using a partial carbon dioxide rebreathing technique: a prospective observational study. Crit Care 2005, 9:R569-R574.

24. Souza R, Amato MB, Demarzo SE, Deheinzelin D, Barbas CS, Schettino GP, Carvalho CR: Pulmonary capillary pressure in pulmonary hypertension. Crit Care 2005, 9:R132-R138.

25. Cannesson M, Besnard C, Durand PG, Bohe J, Jacques D: Relation between respiratory variations in pulse oximetry plethysmographic waveform amplitude and arterial pulse pressure in ventilated patients. Crit Care 2005, 9:R562-R568.

26. Wang XR, Zheng YJ, Tian J, Wang ZH, Pan ZY: A preliminary study on the monitoring of mixed venous oxygen saturation through the left main bronchus. Crit Care 2005, 10:R7.

27. Abrahamson SD, Canzian S, Brunet F: Using simulation for training and to change protocol during the outbreak of severe acute respiratory syndrome. Crit Care 2005, 10:R3.

28. Needham DM, Dennison CR, Dowdy DW, Mendez-Tellez PA, Ciesla N, Desai SV, Sevransky J, Shanholtz C, Scharfstein D, Herridge MS et al.: Study protocol: the Improving Care of Acute Lung Injury Patients (ICAP) study. Crit Care 2005, 10:R9. 